\title{
Cultura bioética y conceptos de enfermedad: el caso House
}

\author{
Bioethical culture and concepts of disease \\ in «House, M. D.»
}

Antonio Casado da Rocha y CRistian SAborido *

Universidad del País Vasco / Euskal Herriko Unibertsitatea

\begin{abstract}
RESUMEN. En la intersección entre la ética aplicada y los estudios sobre divulgación científica en los medios, este trabajo propone un concepto de «cultura bioética» y lo aplica a la exitosa serie de televisión House. Nuestro análisis de la serie muestra la relación existente entre la tensión entre autonomía del paciente y paternalismo médico presente en la teoría bioética y la práctica asistencial, por un lado, con el debate abierto en filosofía de la medicina entre posiciones naturalistas y normativistas sobre los conceptos de salud y enfermedad, por el otro. Se propone también una ampliación del concepto de autonomía que permita avanzar en la resolución de esta tensión, tanto a nivel teórico como práctico.
\end{abstract}

Palabras clave: Bioética, enfermedad, normativismo, naturalismo, autonomía, House.

\section{Introducción}

Todos somos pacientes en potencia. ¿Cómo desearíamos que fuesen nuestros hospitales? ¿Hay divergencias entre nuestras ideas sobre la salud y la enfermedad, y las de los profesionales que allí nos atienden? ¿Cómo incide la filosofía moral en la ética de las profesiones asistenciales? ¿Qué relación hay entre la bioética y la filosofía de la medicina?
ABstract. In between applied ethics and cultural studies of popular representations of science in the media, this paper proposes a concept of «bioethical culture» and applies it to House, M.D., the successful TV medical drama. Our analysis of the series shows that the current tension between paternalism and autonomy - both in bioethical theory and health care practice - is related to an on-going argument in philosophy of medicine between naturalist and normativist concepts of health and disease. We argue for an expansion of the concept of patient autonomy so that this tension might be partly resolved in theory and in practice.

Key words: Bioethics, disease, normativism, naturalism, autonomy, House.

Para responder a estas preguntas es necesario acercar a la filosofía moral el debate sobre los conceptos de salud y enfermedad, que inauguró la filosofía de la medicina como disciplina académica (Engelhardt 1976) y que sigue siendo uno de los más activos en filosofía de la ciencia. Aunque pueden encontrarse precedentes interesantes en la literatura médica (véase por ejemplo los recogidos en Cohen 1955), el debate filosófico con- 
temporáneo se inició propiamente con un artículo en la revista Philosophy of Science (King 1954) y la contribución más citada en este ámbito es la de Christopher Boorse (1975), quien defendió una noción puramente descriptiva de salud como funcionamiento estadísticamente normal. Esta definición ha recibido muchas objeciones (Cooper 2002a), y antes del artículo de Boorse ya se criticaba que los fenómenos patológicos se consideren idénticos a los fenómenos normales respectivos salvo por determinadas variaciones cuantitativas (Canguilhem 1966). Otros autores han criticado la tesis de la neutralidad axiológica del concepto de enfermedad (Engelhardt 1996), defendiendo el carácter intrínsecamente cultural y político de la clasificación de enfermedades; ejemplares en esta línea son los primeros trabajos de Foucault (1961), en los que se ataca la asimilación moderna de la locura a la categoría de enfermedad mental. 1

Es difícil entender la medicina en el siglo XXI sin partir de la constatación, aunque sea muy simplificada, de que entre el XIX y el XX se desarrolló una revolución tecnocientífica que alteró por completo las relaciones tradicionales entre médico y paciente. Tal vez convenga recordar, al respecto, que la medicina fue considerada en la Antigüedad clásica como un arte que con la ayuda de las ciencias teóricas tenía como fin producir la salud de un organismo viviente. A partir de la Ilustración la medicina entra en una fase experimental (cuya figura más representativa sería la de Claude Bernard) en la que se consolida la medicina moderna y se transforma la relación entre profesionales y usuarios de los servicios de salud — que aquí llamaremos «relación asistencial»— principalmente por la mediación de la técnica. Esta transformación se agudizó en la última década del siglo XX, el período de los últimos veinti- cinco siglos en que la relación asistencial ha cambiado más profundamente (Lázaro y Gracia 2006). Coincidiendo con la aparición e institucionalización de la bioética en ese período, la medicina occidental sufrió una nueva transformación, digamos que «postmoderna», en la cual aún estamos inmersos y donde aparecen nuevas formas de entender la relación asistencial, como en la llamada «medicina del deseo» (González Quirós y Puerta 2009), en la que la demanda de servicios sanitarios se incrementa y dirige a fines tradicionalmente no contemplados por la medicina, como los estéticos, los deportivos o los reproductivos. En este trabajo nos proponemos analizar algunos rasgos de esa situación actual de la medicina mediante el estudio de nuestra cultura bioética global y el papel que en ella juegan las narraciones audiovisuales, por un lado, y los conceptos de enfermedad, por el otro.

\section{¿Por qué House? Cultura bioética y televisión}

Antes del siglo XIX, las categorías diagnósticas estaban basadas fundamentalmente en lo que los pacientes contaban a sus médicos. Pero con la consolidación del modelo patoanatómico, esos síntomas pasaron a un segundo plano y las enfermedades fueron reclasificadas de acuerdo con las lesiones descubiertas en estudios anatómicos que empleaban diversas tecnologías para encontrar la «verdad» objetiva de la enfermedad visibilizándola en el interior del cuerpo del paciente, al margen de su experiencia subjetiva (Leder 1992, 96). Este proceso fue descrito por Michel Foucault en su libro El nacimiento de la clínica (1963), donde nos presenta una «arqueología» de la mirada [regard $]$ médica que identifica la racionalidad médica moderna con «el dominio de la mirada atenta, de esta vigilancia empírica abierta a la evidencia de los úni- 
cos contenidos visibles» (Foucault 1963, 6). Que el acto médico por excelencia consista en una mirada viene apoyado por la persistencia de locuciones como «el doctor le verá enseguida», pero no tenemos espacio aquí para adentrarnos en la teoría foucaultiana de la medicina, un tema que además ha recibido ya la debida atención en Isegoría (Novella 2009). En este trabajo señalamos la importancia de esa visión en una serie televisiva como House, que nos presenta la medicina precisamente como una actividad que busca «visualizar» la enfermedad, intentando «apartar» al paciente para centrarse en los signos acumulados por la enfermedad en él (y que en la serie son tantos que el paciente acaba por parecer todo un palimpsesto). Así, en la medicina moderna «el paciente es un hecho exterior en relación a aquello por lo cual sufre; la lectura del médico no debe tomarlo en consideración sino para meterlo entre paréntesis» (Foucault 1963, 23).

Aunque en castellano esta serie apenas ha sido estudiada, al menos desde el punto de vista de la ética (Casado 2009), en inglés existe ya bastante literatura sobre los problemas filosóficos planteados por la serie y un buen estado de la cuestión puede encontrarse en el libro House and Philosophy (Jacoby 2008). Uno de los artículos más interesantes de toda esa literatura (Rich et al. 2008) sostiene precisamente que el célebre Dr. House es el médico moderno por antonomasia, aquel que en los paradigmas anteriores de la medicina controlaba la relación asistencial, pero cuya autoridad ha mermado considerablemente en el escenario actual. Tal como ha explicado Enrique Baca (2006), el sistema sanitario de los países occidentales está caracterizado por la presencia de al menos cuatro agentes, cada uno con su propia «lógica»: a los papeles del médico y del paciente en la relación asistencial hay que añadir el jugado por el «tercer pagador» (ya sea un servicio público de salud o una aseguradora privada) y por la industria farmacéutica. El entorno de House está condicionado por todos esos factores, de manera que para hacer posible su encuentro con la enfermedad el doctor House debe disputar su autoridad - a menudo bien groseramente - con el propio paciente o sus familiares, con otros médicos, incluyendo sus compañeros de trabajo o su jefa, y con otras fuentes de autoridad, como la policía o los códigos religiosos de una sociedad plural (Rich et al. 2008, 227).

Como veremos en este trabajo, a la medicina modernista de House le corresponde un concepto de enfermedad que, siguiendo los términos del debate en cierta filosofía de la medicina, podemos denominar «naturalista». ${ }^{2} \mathrm{Y}$ al entorno médico postmoderno de House le corresponde un concepto que, de la misma manera, podemos denominar «normativista». Estos conceptos tienen relación directa con los aspectos éticos de la relación asistencial; de hecho, muchos de los conflictos que animan la serie están basados en el choque entre conceptos naturalistas y normativistas de la enfermedad o, dicho de otra manera, entre el médico moderno y su entorno postmoderno.

Para abordar estos problemas en un contexto global examinaremos el papel de lo que llamaremos «cultura bioética» en la transmisión de conceptos sobre la enfermedad por vía de narraciones audiovisuales como House. Entendemos la cultura bioética como el conjunto de presunciones y expectativas, a menudo poco reflexivas o inarticuladas (es decir, imágenes o clichés), que tenemos acerca de cómo es o debería ser la relación que a diario se establece entre profesionales y usuarios de los servicios de salud. En general, la cultura bioética incluye presuposiciones en relación con todas las ciencias de la vida, pero en este trabajo nos 
ceñiremos únicamente a la medicina y las profesiones sociosanitarias, es decir, a la común acepción de bioética como ética clínica o asistencial. ${ }^{3}$

Como indica José Antonio Seoane (en Casado 2008a, 13-17), podemos definir la relación asistencial como una práctica social institucionalizada: una actividad humana cooperativa en pro del cuidado de la salud. Al igual que cualquier otra práctica humana, la relación asistencial está guiada por diversas normas, pero no se gobierna únicamente con ellas, sino que también es dirigida por la cultura bioética presente en su entorno: el «clima moral» que impregna las interacciones entre los usuarios y los profesionales en un centro asistencial. Este clima está caracterizado por aspectos lingüísti$\cos$ y comunicativos, hermenéuticos y narrativos, y hasta ejemplares o modélicos; no puede ni debe sustituir a la deliberación colectiva y la prudencia individual para discernir cómo actuar en cada situación particular, pero sí condiciona y moldea su ejercicio.

La cultura bioética contemporánea tiende a ser intercultural y aconfesional, y para ello rehúye las cuestiones de fundamentación y pone más énfasis en la aplicación, buscando justificarse por sus resultados o consecuencias. Como veremos a continuación, este carácter pragmático es especialmente visible en sus manifestaciones en la cultura popular contemporánea. En buena parte, nuestra cultura bioética es una cultura globalizada, audiovisual y de masas; una cultura que ya no es fundamentalmente literaria y que se transmite de manera informal mediante el consumo masivo de entretenimiento televisivo a lo largo y ancho del mundo actual. Tan es así, que el American Journal of Bioethics ha publicado recientemente un artículo sobre los hábitos televisivos de estudiantes de medicina y enfermería en la universidad Johns Hop- kins (Czarny et al. 2008). El trabajo concluye que la gran mayoría de los estudiantes sigue las series ambientadas en el mundo sanitario, y que las más vistas son House y Anatomía de Grey ${ }^{4}$. Seguramente, si el estudio fuese replicado en cualquier otro país no arrojaría resultados muy divergentes. Un estudio de la agencia Eurodata TV Worldwide reveló que House había sido la serie televisiva más vista en todo el mundo en 2008, con un promedio de 82 millones de espectadores en 66 países. ${ }^{5}$

Gregory House es el protagonista de la serie homónima creada por David Shore en 2004 y emitida desde entonces por las televisiones de todo el mundo: un médico polémico e irreverente pero efectivo, que no se fía de nadie y mucho menos de sus pacientes, con quienes no suele ser amable, aunque siempre acaba por descubrir la verdad acerca de sus raras dolencias y casi siempre consigue salvar la vida al paciente. Preguntada por el éxito de la serie, una de sus protagonistas explicaba en El País (03/10/2009) que, como «nos sentimos tan vulnerables ante la muerte», «nos gusta ver a gente peleando con ella en el cine y en la televisión». Parece pues que el doctor House está firmemente instalado en nuestra cultura bioética, y que en ella se le concibe fundamentalmente como un luchador contra la enfermedad y la muerte, clara caracterización del profesional médico moderno.

Ahora bien, no podemos ignorar que el escenario de House no se parece en casi nada a la sanidad real. Algunos comentaristas han criticado la ausencia de personal de enfermería en estas series (Ward y Summers 2008) y la confusión de especialidades que presentan, mixturando el rol del médico de urgencias con el de otros profesionales sanitarios. Pero puede que el éxito de la serie resida precisamente en esa lejanía respecto de la 
realidad, tanto norteamericana como española. Proporcionaría así una fantasía escapista, una evasión de esa «insatisfacción colectiva subyacente» detectada por Moure (2007) y que apenas «queda disimulada por el componente vocacional» de las profesiones asistenciales. El personal sanitario trabaja en condiciones difíciles, expuestos a situaciones de gran sufrimiento y a menudo con resultados frustrantes. No pueden hacer lo que quieren y en ocasiones ni siquiera consiguen hacer lo que deben. No es de extrañar la fascinación que provoca House, un personaje que campa a sus anchas por el hospital sin el menor respeto hacia la burocracia, y que además casi siempre se sale con la suya. En cierto sentido, podría ser que en nuestra cultura bioética todavía es tan fuerte el cliché del «buen doctor» que habría provocado la emergencia de su «sombra», representada por este médico sin escrúpulos, egocéntrico y atormentado, pero genial.

No obstante, a pesar de este carácter ficticio e irreal de la producción televisiva sería un error minusvalorar lo que nos dice el éxito de House en nuestra sociedad actual. Por un lado, es innegable que estas series nos informan acerca de los conocimientos médicos de su época, y no sólo en los aspectos técnicos, sino también en los éticos. Por ejemplo, el discurso de House sobre la dignidad de la muerte en el primer episodio de la serie está tomado de la introducción del libro Cómo morimos, del médico Sherwind Nuland (1995). Por otra parte, la serie ilustra toda una clase de conflictos típicos en la bioética contemporánea, una vez que el médico ya ha perdido la exclusividad del conocimiento acerca del bien del paciente (Veatch 2000). En efecto, vivimos en una cultura bioética en la que, como ya explicó Freud, ni los legos «lo son tanto como pudiera creerse ni los médicos son tampoco aquello que debiera esperarse que fueran y en lo que podrían fundar sus aspiraciones a la exclusividad»» (Freud 1926, 245). Pero lo más importante, a nuestro juicio, es que la serie ilustra también lo que se encuentra a la base de esos conflictos: la tensión entre dos conceptos de enfermedad, dos maneras radicalmente opuestas de entender lo que es ser/estar enfermo. Pasamos a presentarlas a continuación.

\section{El concepto de enfermedad y el debate naturalistas/normativistas}

Aunque la vieja definición de la salud según la OMS como bienestar completo «en lo físico, lo psicológico y lo social» haya sido muy criticada (y con buenas razones: véase por ejemplo Camps 2001, 99), si nos olvidamos del «bienestar completo» aún podemos aprovechar su carácter triádico, la inclusión de tres aspectos esenciales a la hora de abordar la enfermedad. ${ }^{6}$ Autores como Azucena Couceiro o Diego Gracia $(1999,25)$ han argumentado que en la relación asistencial no hay sólo dos partes, y en sus obras y labor docente aparece de manera más o menos explícita un modelo triangular con la persona usuaria, la profesional sanitaria y la sociedad en sus vértices. Este modelo se puede relacionar con un enfoque de la enfermedad que la concibe como una realidad con tres dimensiones (disease/illness/sickness), como una tríada, en la que los tres vértices del «triángulo asistencial» llevan asociado un concepto de enfermedad propio: correspondería a disease la visión profesional de la enfermedad, la evaluación de los eventos negativos según el criterio del personal sociosanitario que atiende al paciente; illness recoge la visión personal de este último, la evaluación de los hechos según la propia perspectiva de la persona enferma; finalmente, el término sickness se asocia a la evaluación de los hechos desde las normas producidas por el Estado y otras organizaciones sociales. 
Esta tríada fue mencionada por primera vez en una tesis doctoral defendida en 1967 (Hoffman 2002) y desde entonces viene siendo utilizada en las humanidades médicas. La tríada refleja diferentes perspectivas profesionales (disease), personales (illness) y sociales (sickness); estas perspectivas describen eventos negativos, cosas malas que nos pasan, y son al mismo tiempo conceptos normativos, que nos invitan a actuar ante la mala suerte o la injusticia que supone enfermar. El concepto personal de enfermedad (illness, que aquí llamaremos EP) es la enfermedad tal como la vive cada uno: una vivencia que reorienta la actividad del sujeto enfermo, lo que a menudo le lleva a comunicar su experiencia subjetiva de ese mal a los demás, y eventualmente le hace pedir ayuda médica. Por otra parte, el concepto profesional o médico de enfermedad (disease, EM) es la enfermedad tal como la ve el personal sanitario: una situación objetivable mediante la ciencia médica que les mueve a identificar esa clase de evento y a cuidar a la persona que lo sufre. Y como un concepto social (sickness, ES), la enfermedad requiere determinar el estatus de la persona enferma, decidiendo quién tiene derecho a tratamiento y ayuda económica y a quién se le pueden disculpar sus obligaciones por hallarse enfermo (en algunos casos, como ciertas enfermedades mentales, es la sociedad la que considera que una persona está enferma, aunque la interesada no lo acepte).

El concepto de enfermedad es, pues, un complejo de «males» (Cooper 2002a) o eventos negativos percibidos por los profesionales asistenciales, sus pacientes y la sociedad. A menudo, la medicina se ha centrado únicamente en EM, definiendo la enfermedad como un fenómeno meramente biológico, y su crítica postmoderna se ha ocupado de ES, insistiendo en la construcción social de la enfermedad (como en la obra de Foucault, ya mencionada al inicio). Con el surgimiento de la bioética (en especial sus versiones más interesadas por la narrativa, como las presentadas en Nelson 1997) y los movimientos por los derechos de los pacientes, la medicina ha recobrado EP, esto es, la fenomenología de la enfermedad tal y como es experimentada por el paciente (cuyo máximo exponente se puede encontrar en Carel 2008).

EP, EM y ES pueden considerarse como funciones que arrojan valores distintos según las circunstancias de cada caso particular. En el modelo típico de relación asistencial, la persona $\mathrm{x}$ siente cierto malestar EP(x) y acude al médico, que le diagnostica una enfermedad particular $\mathrm{EM}(\mathrm{x})$ y pone en marcha ciertos mecanismos de reconocimiento de ese estado, como por ejemplo una baja laboral, ES(x). Todo esto, naturalmente, supone un complejo conjunto de valoraciones biopsicosociales, pero no tiene por qué generar problemas éticos. Sí los hay, no obstante, cuando las diferentes visiones no coinciden; cuando, por ejemplo, la visión personal de la enfermedad no se corresponde con la profesional o la social, cuando la visión del profesional no se ajusta a lo que el paciente o su entorno social consideran adecuado, o cuando la visión de la sociedad está radicalmente en desacuerdo con la vivencia de la enfermedad o la discapacidad tal como la experimentan y elaboran los pacientes y sus cuidadores. ${ }^{7}$

El actual debate filosófico en torno a las nociones de salud y enfermedad suele presentarse como una discusión entre naturalistas y normativistas (Kovács 1998). Por un lado, los naturalistas describen estos conceptos como nociones teóricas objetivas de ciencias como la farmacología o la fisiología, por lo que se fijan exclusivamente en un concepto profesional o biológico de enfermedad (EM). Para los 
normativistas, en cambio, esta dicotomía salud/enfermedad está plagada de prejuicios que poco tienen que ver con una presunta objetividad científica y mucho con todo un bagaje de valores y creencias subjetivas (EP) y sociales (ES) que se debe tener en cuenta (Nordenfelt 1987, Culver y Gert 1982).

Como ya hemos apuntado, el autor más relevante dentro del enfoque naturalista es Boorse $(1975$; 1977; 1997). Al igual que para el resto de teóricos naturalistas, para Boorse la distinción conceptual entre salud y enfermedad hace referencia a otro concepto que consideran objetivo y axiológicamente neutro: el concepto de función biológica. Así, la enfermedad sería la «incapacidad para llevar a cabo todas las funciones fisiológicas típicas con, al menos, un nivel típico de eficiencia» (Boorse 1977, 542). Salud es equivalente a «funcionamiento normal», y aquí «normal» significa «típico», «usual», «mayoritario» en un sentido estadístico. Para Boorse, el funcionamiento sano se corresponde con el «diseño de la especie» y este diseño se puede deducir estadísticamente de las características del conjunto de los organismos de una misma clase de referencia (esto es, el conjunto de individuos que comparten especie, sexo y edad). Aquello que es más común entre los individuos de una misma clase es lo que se corresponde con su diseño específico, lo que equivale a decir que es lo normal y, por lo tanto, lo sano. ${ }^{8}$

Esta propuesta ha sido ampliamente discutida y son muchos los contraejemplos que se pueden encontrar en la literatura especializada (Kovács 1998; Khushf 1997). Por ejemplo, el caso de las enfermedades ampliamente extendidas, como la caries; o el caso de comportamientos funcionales anormales que, sin embargo, no se considera que conlleven una enfermedad para el organismo. Podemos considerar, por ejemplo, el caso de la minoría de indígenas polinesios o precolombinos que no murieron al contacto con los nuevos patógenos infecciosos que introdujeron en sus poblaciones los primeros europeos (Dubos 1987). En una situación en la que la mayoría de los miembros de una clase de referencia mueren o padecen graves sufrimientos, ¿podemos considerar enfermos precisamente a aquellos individuos que, de forma «anormal», no lo hacen?

Además, la misma idea de «función normal» que utiliza Boorse es muy discutible. Este tipo de análisis teórico se sustenta en el presupuesto de que existe una forma unívoca de funcionar para los miembros de una misma clase de referencia. Boorse llega a afirmar incluso que si existe alguna diferencia en el modo de funcionar entre los miembros de una misma clase, esta diferencia es lo suficientemente insignificante, en términos estadísticos, que podemos perfectamente ignorarla (Boorse 1997, 39).

No obstante, esta postura -que Amundson (2000) califica de «determinismo funcional»- realmente no tiene una buena fundamentación biológica. No tenemos pruebas empíricas para creer que las funciones biológicas de los miembros de una especie con el mismo sexo y la misma edad se llevan a cabo de un modo lo suficientemente uniforme como para que podamos distinguir mediante un criterio estadístico a «los normales». La realidad es más bien lo contrario. La variación funcional, que proviene tanto de diferencias genéticas como en el proceso de desarrollo individual de cada organismo, es ubicua en la naturaleza. Y esto es así incluso entre los miembros de una misma «clase de referencia». Del mismo modo que dentro de los individuos de una misma «raza» hay una gran diversidad biológica, dentro de una misma clase de referencia existe una gran variedad de modos de funcionamiento (Amundson 2000, 36). 
No es cierto que, como sugiere la célebre metáfora del reloj de Paley, todas las partes de un organismo estén específicamente diseñadas para funcionar de una determinada manera. Los miembros de una misma clase de referencia no son, ni pueden ser, funcionalmente idénticos. ${ }^{9}$ Diferentes genotipos provocan variedad funcional, pero incluso genotipos idénticos pueden dar lugar, mediante diferentes procesos de desarrollo, a fenotipos funcionalmente distintos. El desarrollo de un organismo permite una cierta plasticidad (Gilbert 1997). Por un lado, unas partes se adaptan a las otras en su crecimiento y, por otra parte, hay una adaptación ontogenética del organismo a las condiciones del ambiente externo. La variación funcional no implica una fisiología normal, sino una fisiología diferente.

La principal crítica que se hace desde muy distintos planteamientos a los posicionamientos filosóficos y éticos basados en este tipo de análisis bioestadístico es que es posible $-\mathrm{y}$ de hecho es algo que ocurre con frecuencia- que individuos de una misma clase, a través de diferentes modos de funcionamiento, alcancen un mismo nivel de eficiencia funcional (Silvers 1998; Cooper 2002b). Por ejemplo, y como señala Amundson $(2000,50)$, dependiendo de las circunstancias una persona en silla de ruedas puede funcionar al mismo nivel o incluso por encima del de una persona con movilidad bípeda, y sin embargo usar una silla de ruedas suele ir asociado al estigma de la enfermedad. No es por tanto posible establecer una diferencia conceptual entre sanos y enfermos que se muestre bien fundada y válida para la práctica biomédica siguiendo el criterio bioestadístico de Boorse.

Sin embargo, hay autores que, como Daniels (1985) o Brock (1993), han defendido que la preservación y restauración de la «función normal» debe ser el objetivo básico del cuidado médico. Se- gún estos autores, el funcionamiento anormal supone necesariamente una disminución de oportunidades para los individuos que lo sufren y, por tanto, la labor del trabajo médico ha de ser la de hacer que los individuos enfermos alcancen la normalidad. La relación asistencial debe basarse por tanto en este tipo de definición biológica (EM) de enfermedad.

Ésta es una perspectiva muy afianzada en la cultura popular y, dentro de su contexto exagerado y poco realista de la ficción televisiva, House puede considerarse como el arquetipo de médico naturalista. Sin embargo, el mismo doctor House se ha de encontrar con muchas de las dificultades e incongruencias a las que lleva este modelo teórico y que ya han sido expuestas por los críticos.

\section{¿Qué concepto de enfermedad transmite House?}

A la luz de las anteriores clasificaciones, puede entenderse al doctor House como un personaje declaradamente naturalista. Siendo como es un personaje centrado en la afirmación de su autoridad cognoscitiva o epistémica, House trata cada enfermedad como un misterio científico que puede y debe resolver, sin importarle las peculiaridades del paciente o los «valores» que puedan afectar a éste. Ya en los primeros capítulos de la serie se destaca, entre sus singularidades como médico, que casi nunca desea hablar con los pacientes; su manera de «verlos» no es verbal. Prácticamente cada capítulo sigue el mismo guión general: una persona aquejada de alguna extrañísima dolencia llega a manos del equipo del Dr. House, quien, después de distintas pruebas, complicaciones e imprevistos, consigue dar con el diagnóstico correcto y con el tratamiento que salva al enfermo. Y todo esto desde una metodología más cercana a la de Sherlock Holmes que a la de un médico moderno habitual (Abrams 2009). 
A House le basta con conocer los síntomas y el historial clínico de sus pacientes para concretar un diagnóstico. Cómo él mismo dice: «nos hicimos médicos para tratar enfermedades. Tratar pacientes es el inconveniente de esta profesión»» (1.1, Piloto). ${ }^{10} \mathrm{Y}$ es precisamente esto, tratar enfermedades, lo que mejor se le da a House; no hay misterio médico que se le resista y (casi) siempre encuentra la solución a tiempo de salvar la vida del paciente. Tratar con las personas se le da notablemente peor. El resto de su equipo, así como sus jefes y compañeros, le respeta y admira en lo profesional aunque no oculten la animadversión que les provoca su difícil personalidad. Al margen de lo anecdótico de algunos rasgos de House - algo ineludible en cualquier narración cuya función primera es entretener al auditorio-, es interesante destacar que su marcado autoritarismo está basado no sólo en su considerable autoridad epistémica (House «sabe»), sino también en su posición privilegiada en la jerarquizada estructura de una organización sanitaria moderna (basada, se diría, en el principio de que «los médicos saben, los enfermeros cuidan y los pacientes obedecen»), lo que le permite trabajar sin tener en cuenta las opiniones de los demás.

Finalmente, su autoridad está basada en la eficacia de su trabajo. House es eficaz porque casi siempre consigue que el paciente sane, pero, ¿qué es lo que se entiende aquí por «sanar»? Consideramos que la idea de salud que subyace a la creencia en la eficiencia profesional de House es similar a la de Boorse y, por lo tanto, es comprensible que entienda la relación asistencial del mismo modo que propugnan teóricos como Daniels o Brock: un enfermo es alguien en el que «algo no funciona bien». El trabajo del médico no es otro sino averiguar qué es eso que no funciona como debería y «arreglarlo», lo que a su vez presupone que ese algo que no funciona puede ser descubierto «desde fuera», sin tener en cuenta cómo lo vive la persona afectada.

Desde este punto de vista, se entiende que House no vea necesario tratar personalmente a sus pacientes, que no le importe lo que ellos piensen acerca de su estado ni lo que deseen con respecto a su tratamiento. House es capaz de engañarlos para conseguir su consentimiento para ciertas pruebas, les oculta información e incluso se burla de sus valores y creencias más profundas si esto le facilita su labor. A veces parece incluso que lo único que motiva a House es encontrar el diagnóstico, la solución al acertijo que representa el enfermo:

- PACIENTE: ¿Quiere hablar de hipocresía? Hable de usted. Va por la vida haciendo como si no le importase nadie y se dedica a salvar vidas.

- HOUSE: Resuelvo enigmas. Salvar vidas es sólo un efecto colateral.

(5.15, Descreido)

El naturalismo de House, en pocas palabras, se ocupa sólo de los hechos; los valores le tienen sin cuidado. Sin embargo, y a pesar de lo que él mismo diga, no sería justo decir que House no se preocupa por beneficiar a sus pacientes. De hecho, es este principio bioético de beneficencia el único al que parece ser sensible, aunque desde su particular forma de entenderlo: beneficiar al paciente consiste, la mayoría de las veces, en librarlo de su enfermedad. El objetivo último de House es el diagnóstico de la enfermedad que, no lo olvidemos, es condición necesaria para acabar con ella (aunque no suficiente, pues hay un pequeño número de casos en la serie en los que la enfermedad diagnosticada no tiene cura) y la enfermedad es algo objetivo y observable científicamente, es decir, «naturalista». El resto de factores «normativistas», como los sentimientos, creencias o deseos del enfermo no tienen importancia, tener que prestar- 
les atención es sólo «el inconveniente de esta profesión». Haya cura o no, el concepto de enfermedad transmitido por la serie está rodeado de una certidumbre epistémica casi total: sólo cuando el propio House se convierte en paciente - en los que posiblemente sean los episodios más interesantes de cada temporada - su imagen escapa de lo que podríamos llamar en términos kuhnianos la «medicina normal», la vigente en los libros de texto de la profesión médica.

En un capítulo en el que House discute sobre el consentimiento para una prueba médica con un familiar de un paciente judío profundamente religioso, expone claramente cuál es la imagen que tiene de sí mismo en relación con los enfermos a su cargo:

- FAMILIAR: Es el cuerpo de mi mujer lo que está enfermo. Su mente y alma están bien.

- HOUSE: Usted vive de acuerdo con 600 compromisos con Dios. ¿Cierto?

- FAMILIAR: En realidad son 613 .

- HOUSE: ¿Los entiende todos?

- FAMILIAR: Bueno, lleva toda una vida aprender...

- HOUSE: Pero los sigue. No los entiende todos pero los sigue porque cree que quien los creó sabía lo que hacía.

- FAMILIAR: ¡Por supuesto!

- HOUSE: Entonces confiará en lo que yo le diga y me permitirá tratar a su mujer porque, en este templo, yo soy el doctor Yahvé.

\section{(4.12, No cambies nunca)}

El autoritarismo de House se construye sobre su autoridad epistémica, sobre su conocimiento de los hechos biológicos. Desde esta perspectiva naturalista, la labor sanitaria es fundamentalmente una lucha entre el médico y la enfermedad, independientemente de cualquier tipo de valoración subjetiva o social acerca de la enfermedad o de la relación asistencial. El médico conoce la forma de curar la enfermedad y, por tanto, conoce «lo que conviene» al paciente. La diferencia pues entre legos y expertos es abismal, y sobre esa distancia se ha construido el paternalismo médico. En esta forma de ver la medicina prima el principio de beneficencia por encima del respeto a la autonomía. Lo más importante es curar la enfermedad porque esto significa beneficiar al paciente, lo quiera éste o no. Aunque podría darse el caso de que un médico fuese naturalista pero no paternalista (ocupándose de los hechos y luego dejando la decisión sobre los valores en manos del paciente), lo habitual es que una cosa lleve a la otra; y, desde luego, hoy por hoy el paternalismo ya no se considera como una forma aceptable en general de entender la relación asistencial (Veatch 2000).

Aunque la idea de que «House es un gran médico porque (casi) siempre sabe lo que hay que hacer» parece ser el mensaje último que nos transmite la serie, no hay un solo episodio en que el naturalismo de su protagonista no conduzca a alguna clase de conflicto ético. House tiene un concepto profesional de enfermedad (EM) que muchas veces difiere de los conceptos profesionales de otros médicos (sus constantes discusiones con su amigo el Dr. Wilson o los enfrentamientos con su jefa administrativa, la Dra. Cuddy, ambos representantes de un profesional sanitario más «normativista», más inclinado a tener en cuenta la perspectiva del paciente, son una buena muestra de ello), y con los otros conceptos personales (EP) o sociales (ES) del paciente y su entorno. Puede apreciarse fácilmente que House suele anteponer la beneficencia a la autonomía del paciente (Stock y Burke 2009, 140-143), tomando muchas veces las decisiones por su cuenta y riesgo y según su propia concepción del bien. Esto da lugar a gran parte de los conflictos morales en la serie, y que surgen de la creciente importancia de la autonomía, algo presente tanto en esta trama televisiva como en las 
relaciones asistenciales reales, con pacientes que cuestionan el conocimiento médico a partir de información que encuentran en Internet (episodio 6.3), etc. En House se pone frecuentemente de relieve la dificultad de conjugar autonomía y responsabilidad en ética asistencial. Por ejemplo, en Que será será (3.06), House intenta convencer a un paciente muy obeso de que su peso constituye una enfermedad aunque él se sienta feliz como es, y lo mismo ocurre en el episodio Feliz pequeña Navidad (3.10) con un familiar de una paciente con enanismo, o en House dividido cuando es incluso capaz de ponerle quirúrgicamente un implante a un joven sordo para que pueda oír sin su consentimiento ni el de su familia. El «Dr. Yahve» sabe lo que hay que hacer, mientras que «el paciente es un ignorante, y su familia es idiota» (5.22). No obstante, no siempre es la visión profesional de House la que se impone y hay algunas excepciones en las que se ve convencido por la concepción social o personal del enfermo, como cuando en Piloto (1.1) permite a una paciente en estado terminal irse a casa para morir sin someterse a más pruebas. House sabe que la paciente podría salvarse si aceptase hacer una prueba más, pero por una vez acepta su decisión (si bien no de manera definitiva).

El hecho de que en algunos episodios House acabe por aceptar posiciones cercanas a las de los normativistas refuerza la hipótesis de que, en la realidad de la práctica asistencial actual, el debate ha de entenderse como un choque, no ya entre naturalismo y normativismo, sino entre el «normativismo débil» y el «normativismo fuerte», tal y como indica Khushf (2007). En su versión débil (a la cual también podríamos llamar «normativismo moderno»), el normativismo considera que los conceptos de salud y enfermedad incorporan valores, pero mantiene una confianza naturalista en que pode- mos distinguir hechos y valores. El normativismo fuerte (o «postmoderno»), por contra, sospecha de todo intento demarcatorio, pues aduce que no se pueden distinguir hechos y valores, y que los factores socioeconómicos condicionan lo que entendemos por patológico.

Sin llegar a abrazar una posición normativista fuerte, sí parece que el naturalismo de House se ha ido complementando y matizando a lo largo de la serie. Esta podría ser la clave de la evolución en su personaje. Tras seis temporadas, podría decirse que House se ha «humanizado»y vuelto más complejo, en parte porque se ha visto obligado a contrastar y matizar su naturalismo inicial con los conceptos de enfermedad más normativistas representados por algunos pacientes y otros profesionales sanitarios. Así, en la última temporada hay todo un episodio (6.14) narrado desde el punto de vista de la Dra. Cuddy, un personaje cuyo cargo en el Hospital le obliga a introducir constantemente los factores socioeconómicos y organizativos en la toma de decisiones. Este carácter postmoderno, ya apuntado en otros episodios, se ha agudizado con pacientes que toman decisiones terapéuticas siguiendo el criterio de los lectores de su blog (6.15).

A nuestro juicio, para comprender mejor esta tensión entre los normativismos débil y fuerte, y la forma en la que ésta se manifiesta en House, es preciso ofrecer una visión de la autonomía algo más amplia de lo que suele ser habitual en ética asistencial - sobre todo en su puesta en práctica-, pues en ese contexto el respeto de la autonomía del paciente a veces se entiende de una manera excesivamente decisional, como la mera opción entre alternativas terapéuticas o paliativas, o su consentimiento más o menos informado. Sin embargo, la autonomía tiene como mínimo dos dimensiones más: una funcional, en la que entran 
las capacidades del paciente para manejarse, para llevar su propia vida (incluyendo la ejecución de su plan de cuidados), y otra informacional, en la que entra el control del paciente sobre la información asociada a su estado de salud (Casado 2008a, 82-83).

Aunque las tres dimensiones de la autonomía aparecen en la serie, en ella también se manifiesta ese indebido protagonismo de la autonomía decisional. Podría haber una explicación histórica: la autonomía decisional, asociada a la medicina crítica y de urgencias, corresponde a un concepto de enfermedad aguda. Como inicialmente la implantación institucional de la bioética tuvo lugar en un contexto de hospital terciario fuertemente tecnologizado - del que el modelo House no es sino una exageración: pocos hospitales habrá tan limpios y tecnológicos como el que vemos en la serie_-, su objetivo inicial fue proporcionar principios para guiar la actuación profesional en situaciones de toma de decisiones de vida o muerte. Pero al final del s. Xx el concepto de paciente sufrió una gran transformación, asumiendo un perfil más «sociosanitario», y la atención de la bioética se desplazó hacia la enfermedad crónica, la medicina familiar y comunitaria y la atención primaria, a largo plazo, allí donde la atención pasa del órgano al paciente como persona (Casado 2008a, 160-164). ${ }^{11}$ Es en ese contexto, que no suele aparecer tanto en la serie de televisión, donde se aprecia que la autonomía del paciente tiene otras dimensiones más allá de la decisional.

En suma, este esbozo de las dimensiones presentes en el concepto de autonomía permite entender por qué, como dice Pablo Simón en su libro sobre el consentimiento informado (2000), un sujeto competente o capaz no necesariamente ha de realizar siempre acciones autónomas: puede faltarle información o sobrarle alguna constricción externa. Además, esta concepción amplia de la autonomía es coherente con la tesis general de ese libro, que plantea que el respeto por la autonomía no es tanto un principio más de la bioética como un radical cambio de perspectiva sobre lo que debe ser la relación asistencial (Simón 2000, 277; 167). Pues, como hemos visto en este artículo, la autonomía no es sólo un atributo de determinadas decisiones, sino también un atributo de las personas: algo que se vive según la cultura bioética de cada comunidad humana.

\section{Conclusiones}

Vivimos en una cultura bioética profundamente influida por el papel de los nuevos medios y productos de entretenimiento audiovisual. En esta cultura, por un lado, el médico es profundamente naturalista y paternalista (y considera que su labor se limita a vencer a la enfermedad), mientras que por el otro el paciente es cada vez más normativista y autonomista (y tiende a identificar su salud con su autonomía). Sin embargo, ni el naturalismo tiene por qué ser paternalista ni el concebir la enfermedad desde el normativismo debería anteponer la autonomía del paciente sobre cualquier otra consideración. Esta tensión, presente en la práctica cotidiana de las relaciones asistenciales, no pone las cosas fáciles a la hora de construir una bioética con pretensiones de universalidad. Pero también tiene consecuencias a nivel teórico. El modelo House está centrado en la medicina crítica, en situaciones de vida-o-muerte; pero cuando la bioética desplaza su foco de atención desde la enfermedad hacia la persona, este desplazamiento tiene profundas consecuencias sobre la manera como debemos entender la relación entre los principios tradicionales de la bioética y otros valores. Entre otras, y para terminar, resaltaríamos la importancia que co- 
bran ahora la redefinición de salud y enfermedad en términos más normativos que naturalistas, con el consiguiente debilitamiento de la distinción entre hechos y valores.

En este trabajo hemos presentado algunos rasgos de la cultura bioética presente en los países desarrollados. A la hora de hacer una aportación a una bioética global e intercultural, en lugar de partir de teorías en conflicto y de alto nivel de abstracción, hemos preferido utilizar una estrategia más inductiva, examinando la práctica real y el clima moral de nuestras instituciones sanitarias, así como las narraciones audiovisuales que configuran nuestras expectativas sobre la relación asistencial. Entre estas narraciones la serie House suscita un considerable interés, tanto en el público general como entre los profesionales asistenciales. Hemos visto cómo, al margen de las licencias poéticas impuestas por las normas del género televisivo, la serie ilustra lo que se encuentra a la base de muchos conflictos en ética asistencial: la tensión entre dos conceptos de enfermedad que otorgan diferente peso a las perspectivas del profesional, por un lado, y a las del paciente y su entorno social, por el otro. Así, la cultura bioética presente en House está caracterizada por la presencia de un médico con un concepto naturalista de la enfermedad en un entorno asistencial postmoderno con un concepto más normativista de la enfermedad. Esta hipótesis se refuerza al considerar la evolución en los temas de la serie, en la que temporada tras temporada hemos asistido a cierta «humanización» del personaje de House, de manera que su planteamiento naturalista inicial se ha ido complementando y matizando con otras perspectivas.
La conclusión principal de este trabajo es que en nuestra cultura bioética coexisten versiones dispares del concepto de enfermedad, y también del concepto de autonomía. Esta disparidad tiene consecuencias teóricas y prácticas. En teoría ética es preciso articular y fundamentar mejor el principio de respeto a la autonomía del paciente, relacionándolo con otras explicaciones de la autonomía moral y tratando de no reducirlo a su dimensión decisional. En la práctica, es necesario adaptar la visión predominantemente moderna de muchos profesionales sanitarios para que puedan desarrollar su labor en un contexto asistencial que ha cambiado mucho en los últimos años, y que requiere de ellos una sensibilidad al concepto normativista de enfermedad que a menudo no está presente en su formación científica.

\section{Agradecimientos}

Los autores agradecen numerosas aportaciones de Arantza Etxeberria Agiriano a una versión anterior de este trabajo, así como los comentarios de un revisor anónimo de Isegoría y de los participantes en el simposio sobre bioética del XV Congreso Internacional de la Asociación Filosófica de México, celebrado el 25-29 de enero de 2010. También agradecen el apoyo del Ministerio de Ciencia e Innovación a través de los proyectos de investigación «El concepto de autonomía en bioética e investigación biomédica: aspectos filosóficos y sociales» (FFI2008-06348-C02-02/ FISO) y «Explicando la organización biológica: entre el mecanicismo y la autonomía» (FFI2008-06348-C02-01/FISO), así como una beca predoctoral del Gobierno Vasco. 


\section{REFERENCIAS}

Abrams, J. (2009). The Logic of Guesswork in Sherlock Holmes and House. En: H. JACOBY (ed.), House and Philosophy: Everybody Lies. Hoboken, NJ: John Wiley \& Sons; pp. 55-70.

Amundson, R. (2000). Against Normal Function. Studies in History and Philosophy of Biological and Biomedical Sciences 31 (1): 33-53.

BACA, E. (2006). Las cuatro «lógicas» del sistema sanitario. Claves de razón práctica 165: 42-47.

Boorse, C. (1975). On the Distinction between Disease and Illness. Philosophy and Public Affairs 5: 49-68.

- (1977). Health as a Theoretical Concept. Philosophy of Science 44(4): 542-573.

- (1997). A Rebuttal on Health. En: J. M. Humber y R. F. Almeder (eds.), What is Disease. Totowa, NJ: Humana Press; pp. 3-134.

Brock, D. W. (1993). Life and Death. Cambridge, MA: Cambridge University Press.

Camps, V. (2001). Una vida de calidad. Reflexiones sobre bioética. Barcelona: Crítica.

Canguilhem, G. (1966). Le normal et le pathologique. Paris: PUF.

CAREL, H. (2008). Illness. Stocksfield: Acumen.

Casado, A. (2008a). Bioética para legos. Una introducción a la ética asistencial. Madrid / México DF: CSIC / Plaza y Valdés.

- (2008b). Inflationary Bioethics: On Fact and Value in the Philosophy of Medicine. Praxis Journal of Philosophy 1(2): 30-40.

- (2009). ¿Qué cultura bioética compartimos legos y profesionales sanitarios? Eidon. Revista de la Fundación de Ciencias de la Salud 29: 16-21.
Cohen, H. (1955). The evolution of the concept of disease. Proceedings of the Royal Society of Medicine 48: 155-160.

Cooper, R. (2002a). Disease. Studies in History and Philosophy of Biological and Biomedical Sciences 33: 263-82.

Cooper, R. (2002b). Can it be a good thing to be deaf? Journal of Medicine and Philosophy 32: 563-583.

Culver, Ch. y Gert, B. (1982). Philosophy in Medicine. New York: Oxford University Press.

Czarny, M. J., Faden, R. R., Nolan, M. T., Bodensiek, E. y Sugarman, J. (2008). Medical and nursing students' television viewing habits: Potential implications for bioethics. American Journal of Bioethics 8(12): 1-8.

DAniels, N. (1985). Just Health Care. Cambridge, MA: Cambridge University Press.

Dubos, R. (1987). Mirage of Health. Utopias, Progress and Biological Change. New Brunswick and London: Rutgers University Press.

ENGELHARDT, H. T., Jr. (1976). Is there a philosophy of medicine? Philosophy of Science Proceedings of the biennial meeting of the philosophy of science association, vol. 1976. Two: Symposia and Invited Papers; pp. 94-108.

- (1996). The Foundations of Bioethics, New York: Oxford University Press, 2. ${ }^{a}$ edición.

Foucault, M. (1961). Folie et déraison: Histoire de la folie à l'âge classique. Paris: Plon; 2. ${ }^{a}$ edición, con nuevo prefacio y apéndices: Gallimard, 1972.

- (1963). El nacimiento de la clínica. Una arqueología de la mirada médica. México DF: Siglo XXI, 1980. 
Freud, S. (1926). La cuestión del análisis profano. En: S. Freud, Esquema del psicoanálisis y otros escritos de doctrina psicoanalítica. Madrid: Alianza, 1984; pp. 244-317.

Gilbert, S. F. (1997). Developmental Biology. Sunderland, MA: Sinauer Associates.

GonzÁlez Quirós, J. L. y Puerta J. L. (2009). Tecnología, demanda social y «medicina del deseo». Medicina Clínica 133(17): 671-675.

Gracia, D. (1999). Planteamiento general de la bioética. En: A. Couceiro (ed.), Bioética para clínicos. Madrid: Triacastela; pp. 19-35.

JACOBY, H. ed. (2008). House and Philosophy: Everybody Lies. Hoboken, NJ: John Wiley \& Sons.

KhushF, G. (1997). Why bioethics needs the philosophy of medicine: some implications of reflection on Concepts of Health and disease. Theoretical Medicine and Bioethics 18: 145-163.

- (2007). An agenda for future debate on concepts of health and disease. Medicine, Health Care and Philosophy 10(1): 19-27.

KING, L. (1954). What is a Disease? Philosophy of Science 21: 193-203.

Kingma, E. (2007). What Is It to Be Healthy? Analysis 67: 128-33.

KovÁcs, J. (1998). The concept of health and disease. Medicine, Health Care and Philosophy 1: 31-39.

LÁZARO, J. y GRACIA, D. (2006). La relación médico-enfermo a través de la historia. Anales del Sistema Sanitario de Navarra 29 (supl. 3): 7-17.

LEDER, D. (1992). The experience of pain and its clinical implications. En: J. L. Peset y D. Gracia (eds.), The ethics of diagnosis. Dordrecht: Kluwer; pp. 95-105.

Moure, E. (2007). ¿Por qué los médicos en España no son como el doc- tor House? El País (27 de marzo), p. 53.

Nelson, H. ed. (1997). Stories and Their Limits. Narrative Approaches to Bioethics. New York: Routledge.

Nervi, M. (2010). Mechanisms, malfunctions and explanation in medicine. Biology and Philosophy 25: 215-228.

Nordenfelt, L. (1987). On the Nature of Health. An Action-Theoretic Approach. Series Philosophy and Medicine, vol. 26. Dordrecht - Boston: D. Reidel Publishing Company.

Novella, E. J. (2009). El joven Foucault y la crítica de la razón psicológica: en torno a los orígenes de la Historia de la locura. ISEGORÍA. Revista de Filosofía Moral y Política 40: 93-113.

Nuland, S. (1995). How We Die: Reflections on Life's Final Chapter. New York: Vintage.

Rich, L. E.; Simmons, J.; Adams, D.; Thorp, S. y Mink, M. (2008). The Afterbirth of the Clinic: a Foucauldian perspective on «House M.D.» and American medicine in the $21 \mathrm{st}$ century. Perspectives in Biology and Medicine 51(2): 220-37.

Silvers, A. (1998). A Fatal Attraction to Normalizing. En: E. Parens (ed.), Enhancing Human Traits: Ethical and Social Implications. Washington, DC: Georgetown University Press.

SIMÓN, P. (2000). El consentimiento informado. Historia, teoría y práctica. Madrid: Triacastela.

Sirgiovanni, E. (2009). The Mechanistic Approach to Psychiatric Classification. Dialogues in Philosophy, Mental and Neuro Sciences 2(2): 45-49.

Stock, B. N. y Burke, T. B. (2008). To Intubate or Not to Intubate: House's Principles and Priorities. En: H. JACOBY (ed.), House and Philosophy: 
Everybody Lies. Hoboken, NJ: John Wiley \& Sons; pp. 137-149.

TAuber, A. I. (2005). Patient Autonomy and the Ethics of Responsibility. Cambridge, MA: MIT Press.

VeATCH, R. M. (2000). Doctor Does Not Know Best: Why in the New Century Physicians Must Stop Trying to Benefit Patients. Journal of Medicine and Philosophy 25(6): 701-721.

WARD, F. R. y Summers, S. (2008). Ethics Education, Television, and
Invisible Nurses. The American Journal of Bioethics 8(12): 15.

WicClair, M. R. (2008). The Pedagogical Value of House, M.D.-Can a Fictional Unethical Physician Be Used to Teach Ethics? The American Journal of Bioethics 8(12): 16-17.

Wulf, H. R., Pedersen, S. A., y RosenBERG, R. (2002). Introducción a la filosofía de la medicina. Madrid: Triacastela.

\section{NOTAS}

* Dirección para correspondencia: Facultad de Filosofía y CC. de la Educación. Tolosa Etorbidea, 70. 20018 Donostia - San Sebastián (España). Correo electrónico: antonio.casado@ehu.es, cristian.saborido@ehu.es.

1 Uno de los pocos libros recientemente traducidos al castellano sobre la materia, la Introducción a la filosofia de la medicina de Wulf, Pedersen y Rosenberg (2002), tiene como tema central la crítica a un concepto biológico de enfermedad del que la obra de Boorse es sólo un exponente (y que remite a otros autores, algunos con un concepto «mecánico» de la enfermedad, y otros con un enfoque que Wulf et al. denominan «naturalista» en el caso de las enfermedades mentales). En cualquier caso, los autores aceptan que el modelo mecánico-naturalista constituye una parte indispensable del concepto de enfermedad, pero se oponen a la pretensión de que ofrezca una descripción completa, y buscan ampliarlo mediante una estrategia «hermenéutica» que les permita «tener en cuenta los problemas que atañen a los valores, la moral y las intenciones» (Wulf et al., 2002, 94-95). Esta ampliación supone de hecho dar la razón a las posiciones no naturalistas (o, como veremos, "normativistas») en el debate sobre los conceptos de salud y enfermedad, conceptos que funcionan como criterios de demarcación para trazar las líneas de separación entre lo que es objeto de la medicina y lo que no lo es. Y éste es un debate que, como señala Khushf (2007), es crucial para entender el futuro de la sanidad.

2 En este trabajo no empleamos el término «naturalismo» como a veces se utiliza en la literatura sobre bioética, sino únicamente en el sentido empleado por Khushf (2007) para caracterizar las posiciones que ven los conceptos de salud y enfermedad como axiológicamente neutros o libres de valoraciones sociopolíticas.

${ }^{3}$ En este paper emplearemos «asistencial» en lugar de «clínica» por dos razones. Primera, porque este término se está volviendo de uso común en España, donde desde los años 90 se han comenzado a crear Comités de Ética Asistencial en la mayoría de los hospitales. Segunda, porque el término es más preciso e incluyente, ya que hay relaciones asistenciales, como por ejemplo algunas de las que se establecen en residencias de ancianos, pisos de acogida de menores, etc., que no tienen por qué ser clínicas.

4 En un comentario a ese artículo, Mark Wicclair (2008) pone en duda que estos resultados sean muy significativos. No deberíamos asumir demasiado rápido que la gente que ve series de televisión lo hace en busca de modelos a seguir. Por lo general, los televidentes buscan entretenimiento y saben que en la vida real no ha de imitarse el comportamiento de un médico que miente, infringe el deber de confidencialidad, insulta a sus jefes y colegas, y se salta el consentimiento informado del paciente. El propio estudio de Czarny y colaboradores señala que sólo un $14 \%$ de los encuestados calificó de «importante» la formación sobre asuntos bioéticos que obtenían de las series hospitalarias. En este tema, las principales fuentes de información siguen siendo lo aprendido en la universidad y mediante artículos científicos, así como las conversaciones con familia y amigos. Y cuando la encuesta preguntó a los estudiantes de medicina de tercer y cuarto curso acerca de si los problemas éticos se resolvían bien en esas series, sólo contestaron afirmativamente en cinco situaciones (equidad en el acceso a servicios sanitarios, final de la vida, calidad de vida, revelación del diagnóstico y distribución de recursos), mientras que en otros nueve escenarios la sensación mayoritaria era que las series no trataban adecuadamente los problemas éticos asociados al transplante de órganos, la confidencialidad, la educación de los profesionales asistenciales, los usos «cosméticos» de la medicina, los experimentos con humanos, las enfermedades infecciosas, el consentimiento informado, los errores médicos y la mala praxis. 
5 Pueden consultarse los datos de audiencia en en.wikipedia.org/wiki/House (TV series).

6 No entraremos ahora en detalles acerca de por qué considerar más útil partir del concepto de enfermedad que del de salud. Baste decir que es una opción metodológica razonable a la luz de ciertos argumentos que sostienen la prioridad epistémica (y hasta ética) del primero sobre el segundo.

7 Por supuesto, este modelo típico es una enorme simplificación y no cubre muchas interacciones asistenciales. Por ejemplo, emitir o no un certificado de virginidad es una cuestión que compete a la ética asistencial, y en la que están involucrados valores religiosos y sociales, pero no es una cuestión que tenga que ver prima facie con la enfermedad. Tal vez podría serlo si para determinada cultura la actividad sexual prematrimonial fuese algo así como una enfermedad social (ES), algo que obviamente no tiene un correlato en la comprensión estrictamente médica (EM), y de ahí el conflicto ético para los profesionales. Lo que queremos decir es que, en general, los problemas éticos suelen darse cuando ES, EP y EM arrojan valores muy divergentes para el mismo caso.

8 En un interesante trabajo reciente de Nervi (2010) se propone un análisis naturalista que considera a los mecanismos patológicos no como mecanismos fisiológicos anómalos, sino como un tipo independiente de mecanismo biológico en el que es posible establecer características y relaciones causales propias. En el terreno más difícil para un abordaje naturalista, el de las taxonomías psiquiátricas, Sirgiovanni (2009) ha propuesto un enfoque de la enfermedad basado en explicaciones mecanicistas multinivel.

9 Elselijn Kingma (2007) señala que incluso el hecho de considerar que los individuos que comparten sexo, edad y especie constituyen una misma «clase de referencia» y no lo hacen en cambio los individuos que comparten otro tipo de propiedades, como la ceguera o el estar afectados de neumonía, ya es una elección que presupone una distinción subjetiva previa acerca de lo que es normal y lo que es patológico.

10 Además de por su título, identificaremos los episodios de House por el número de temporada seguido del correspondiente al episodio.

11 Tal como ha sugerido Diego Gracia en sus últimos trabajos, que el «lenguaje de los valores» se revele más adecuado que el de los principios podría ser otra prueba de que estamos ante un proceso de «inflación» o extensión de la bioética, en la que ésta va más allá de la «ética mínima» y pone en cuestión los modelos tradicionales de la autonomía del paciente y la responsabilidad profesional (Casado 2008b). 